\title{
A dual-quenched ECL immunosensor for ultrasensitive detection of retinol binding protein 4 based on luminol@AuPt/ZIF-67 and $\mathrm{MnO}_{2} @ \mathrm{CNTs}$
}

Wei Gong ${ }^{1,2 \dagger}$, Suqing Yang ${ }^{3 \dagger}$, Fen Zhang ${ }^{1,2}$, Fengshun Tian ${ }^{1,2}$, Junman Chen ${ }^{4}$, Zhigang Yin ${ }^{4}$, Shijia Ding ${ }^{4}$, Wei Yang ${ }^{4^{*}}$ and Rong Luo ${ }^{1,2^{*}}$

\begin{abstract}
Background: Retinol binding protein 4 (RBP4) has been regarded as an important serological biomarker for type 2 diabetes mellitus (T2DM). Hence, the construction of a highly sensitive detection method for RBP4 is the key to early prevention and multidisciplinary intervention of T2DM. In this work, a dual-quenched electrochemiluminescence $(E C L)$ immunosensor has been fabricated for ultrasensitive detection of RBP4 by combining zeolitic imidazolate framework-67/AuPt-supported luminol (luminol@AuPt/ZIF-67) with $\mathrm{MnO}_{2}$ nanosheets-grown on carbon nanotubes ( $\left.\mathrm{MnO}_{2} @ \mathrm{CNTS}\right)$.

Results: AuPt/ZIF-67 hybrids with high-efficiency peroxidase-like activity could provide multipoint binding sites for luminol and antibodies and significantly boost the amplified initial signal of the ECL immunosensor. Upon glutathione/ $\mathrm{H}_{2} \mathrm{O}_{2}$ coreactants system, $\mathrm{MnO}_{2} @ C N T s$ composites could quench the initial signal by inhibiting mimic peroxidase activity of luminol@AuPt/ZIF-67. Moreover, the absorption spectrum of the $\mathrm{MnO}_{2} @ C N T s$ composites completely overlaps with the emission spectrum of luminol, which can further reduce initial signal by ECL resonance energy transfer (ECL-RET).
\end{abstract}

Conclusions: Benefiting from the above-mentioned properties, the designed immunoassay sensitivity exhibited excellent sensitivity and relative stability for RBP4 detection range from 0.0001 to $100 \mathrm{ng} \mathrm{mL}^{-1}$ with a low detection limit of $43 \mathrm{fg} \mathrm{mL}^{-1}$. Therefore, our ECL immunosensor provides an alternative assaying strategy for early diagnosis of T2DM.

Keywords: Luminol, Zeolitic imidazolate framework-67, $\mathrm{MnO}_{2}$ nanosheets, Electrochemiluminescence resonance energy transfer, Retinol binding protein 4

*Correspondence: yang623621810@163.com; luorongy@163.com tWei Gong and Suqing Yang contributed equally to this manuscript ${ }^{1}$ Medical Examination Centre, The First Affiliated Hospital of Chongqing Medical University, Chongqing 400016, China

${ }^{4}$ Key Laboratory of Clinical Laboratory Diagnostics (Ministry

of Education), College of Laboratory Medicine, Chongqing Medical University, Chongqing 400016, China

Full list of author information is available at the end of the article

\section{Background}

Type 2 diabetes mellitus (T2DM) is a pyramidally widespread multifactor disease with severe life-threatening complications including cardiovascular disease (CVD), heart failure, and chronic kidney disease (CKD) [1, 2]. According to Global Report on Diabetes of WHO in 2016 and The Berlin Declaration in 2018, early detection and proper multidisciplinary interventions are permits use, sharing, adaptation, distribution and reproduction in any medium or format, as long as you give appropriate credit to the original author(s) and the source, provide a link to the Creative Commons licence, and indicate if changes were made. The images or other third party material in this article are included in the article's Creative Commons licence, unless indicated otherwise in a credit line to the material. If material is not included in the article's Creative Commons licence and your intended use is not permitted by statutory regulation or exceeds the permitted use, you will need to obtain permission directly from the copyright holder. To view a copy of this licence, visit http://creativecommons.org/licenses/by/4.0/. The Creative Commons Public Domain Dedication waiver (http://creativeco mmons.org/publicdomain/zero/1.0/) applies to the data made available in this article, unless otherwise stated in a credit line to the data. 
the priority among priorities of T2DM [3, 4]. Retinol binding protein 4 (RBP4), an insulin resistance-related hormone, is a potential serological biomarker for early monitoring and diagnosis of T2DM [5-7]. However, conventional RBP4 quantitative detection methods, such as enzyme immunoassay (EIA) [8], enzyme linked immunosorbent assay (ELISA) [9], and radioimmunoassay [10], usually suffer from insufficient sensitivity, expensive experimental facilities and complicated operation procedures. Thus, in order to address the aforementioned challenges, establishing effective RBP4 analysis strategy with the advantages of easy manipulation, high sensitivity, and low cost remains urgent requirement for modern clinical diagnosis of T2DM.

Metal organic frameworks (MOFs) as a novel kind of nanomaterial-based artificial enzymes, possess superior biocompatibility, high catalytic activity and durability which demonstrate great potential to replace biological enzymes as high-efficiency catalysts [11-13]. Zeolitic imidazolate frameworks (ZIFs), an attractive subclass of MOFs, have received considerable attention in the bio-analysis applications ascribing to their unique characteristics of large specific surface area, adjustable structure and ordered crystalline pores [14]. More importantly, the imidazolate linkers, especially the imidazole nitrogen site within the porous frameworks, endow ZIFs with excellent selective adsorption properties [15] and catalytic activities [16], making them good candidates in the field of electrochemical sensing.

Furthermore, multi-layered MOFs nanozymes, especially coordinating MOFs with noble metal (Au and Pt) nanoparticles (NPs) demonstrate several significant advantages [17]. For instance, the incorporation of highly dispersed Pt NPs with modulated electronic configuration on a surfactant-free MOF surface has been used to deliver decent electrocatalytic activity under both alkaline and acidic circumstances and promote the electrochemical hydrogen evolution reaction (HER) [18]. More importantly, the introduction of noble metal NPs also can be employed as powerful support matrixes to anchor more molecular luminophores (luminol or its derivatives) by covalent bonding, thus improving the analytical performance of electrochemiluminescence (ECL) biosensor [19, 20]. Inspired by the above research, ZIF-67-supported AuPt bimetallic NPs (AuPt NPs) and luminol nanozymes (luminol@ AuPt/ZIF-67) have been synthesized for the first time. By combining the merits of ZIF-67 and AuPt NPs, this composite material not only ensures uniform dispersion of AuPt NPs and luminol but also provide larger surface area to load more antibodies, thus greatly enhancing luminescence efficiency through peroxidaselike activity of ZIF-67.
Additionally, seeking highly efficient quenchers to quench the luminophores is another crucial challenge in ECL immunoassay. The two-dimensional (2D) manganese dioxide $\left(\mathrm{MnO}_{2}\right)$ nanosheets possess high energy density, ecological friendliness and non-toxic properties, which are regarded as one of the most promising high-performance ECL quenchers [21, 22]. Zhang's group utilized large Pt NPs as the nanocarrier for $\mathrm{MnO}_{2}$ nanosheets to realize quantitative detection of glutathione (GSH), in which $\mathrm{MnO}_{2}$ nanosheets could react with GSH to generate $\mathrm{Mn}^{2+}$, thus scavenging reactive oxygen species (ROSs) properties and inhibiting mimic peroxidase activity of nanozymes [23, 24]. Moreover, the emission spectrum of luminol $(\sim 425 \mathrm{~nm})$ [25] overlaps the absorption spectrum of $\mathrm{MnO}_{2}$ nanosheets (300$600 \mathrm{~nm}$ ) [26] completely, enabling the efficient ECLresonance energy-transfer (ECL-RET) to diminish ECL intensities of luminol [27]. This dual-quenching property endows $\mathrm{MnO}_{2}$ with superior performance, which is however, also relate to its size and dispersion $[28,29]$. Hence other nanomaterials are commonly used to modify $\mathrm{MnO}_{2}$ to enhance their catalytic ability as quenchers. Abundant works have reported that carbon nanotubes (CNTs) with various extraordinary properties including unique nano-size, high specific surface area, superior adsorption effect and multifunctional binding sites, have been generally developed as supporting nanomaterials to modify metals, metal oxides and other materials [30-32]. The excellent modification ability and compatibility of carbon nanotubes for other nanomaterials may significantly improve the electrical conductivity, dense morphology and dispersion of $\mathrm{MnO}_{2}$. Therefore, the combination of CNTs and $\mathrm{MnO}_{2}$ to construct high-efficiency quenchers is the key to further broaden their application in ECL immunoassay.

Herein, a dual-quenched ECL-RET immunosensor was established for ultrasensitive detection of RBP4 based on luminol@AuPt/ZIF-67 nanozymes and $\mathrm{MnO}_{2} @$ CNTs quenchers. As illustrated in Scheme 1A, AuPt/ ZIF-67 was stepwise synthesized by a simple wet-chemical method. Subsequently, luminol was combined with AuPt/ZIF-67 through covalent bonding between AuPt NPs and the amino groups of luminol to form luminol@AuPt/ZIF-67 hybrids as ECL donors. As shown in Scheme $1 \mathrm{~B}$, by growing $2 \mathrm{D} \mathrm{MnO}_{2}$ nanosheets on the surface of CNTs, the high-performance $\mathrm{MnO}_{2} @ \mathrm{CNTs}$ quenchers are in situ synthesized as the ECL acceptor and then conjugated with RBP4 secondary antibodies $\left(\mathrm{Ab}_{2}\right)$ through electrostatic effect, forming $\mathrm{Ab}_{2}-\mathrm{MnO}_{2} @$ CNTs composites. As displayed in Scheme 1C, the ECL immunosensor was prepared by incubating RBP4 monoclonal antibody $\left(\mathrm{Ab}_{1}\right)$ on the glassy carbon electrode (GCE) modified with luminol@AuPt/ZIF-67 hybrids. In 


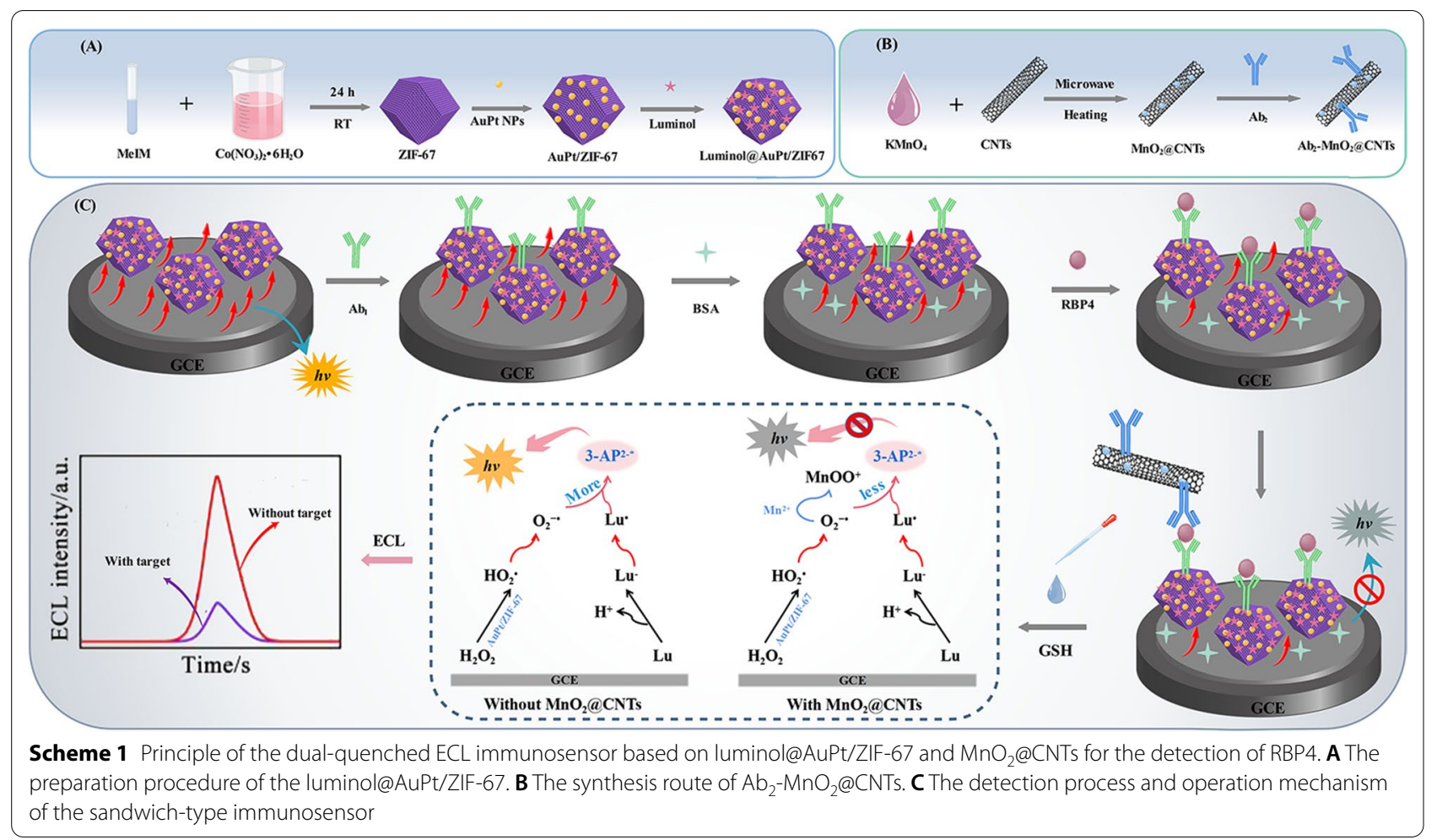

the presence of $\mathrm{RBP} 4$, the $\mathrm{Ab}_{2}-\mathrm{MnO}_{2} @ \mathrm{CNTs}$ bioconjugates were immobilized on the surface of luminol@ AuPt/ZIF-67 decorated electrode based on a sandwichlike immuno response. Meanwhile, GSH was introduced into the system as a reducing agent to constructed a dualquenched immunosensor according to the ECL-RET and consumption of ROSs by $\mathrm{Mn}^{2+}$, which successfully achieved highly sensitive enzyme-free detection of RBP4.

\section{Methods}

\section{Preparation of luminol@AuPt/ZIF-67 hybrids}

ZIF-67 particles was synthesized in accordance with the method reported previously [33]. Typically, $0.364 \mathrm{~g}$ of $\mathrm{Co}\left(\mathrm{NO}_{3}\right)_{2} \cdot 6 \mathrm{H}_{2} \mathrm{O}$ and $0.41 \mathrm{~g}$ of MeIM was dispersed in $20 \mathrm{~mL}$ methanol solution, respectively. The former pink solution was added into the colorless solution under vigorous stirring. The mixture was treated with ultrasound for about 1 min and kept static at room temperature (RT) overnight. The sediment with purple color was collected by centrifugation $(9000 \mathrm{rpm}, 5 \mathrm{~min}$ ) and then washed with methanol five times.

The AuPt/ZIF-67 hybrids preparation was shown in Scheme 1A. The previously synthesized ZIF-67 was added into $20 \mathrm{~mL}$ methanol solution and stirred for $25 \mathrm{~min}$ to acquire a uniformly dispersed solution. After that, $0.5 \mathrm{~mL}$ of $\mathrm{HAuCl}_{4} \cdot 6 \mathrm{H}_{2} \mathrm{O}(1.0 \mathrm{wt} \%)$ and $0.5 \mathrm{~mL}$ of $\mathrm{H}_{2} \mathrm{PtCl}_{6} \cdot 6 \mathrm{H}_{2} \mathrm{O}(1.0 \mathrm{wt} \%)$ were added to the
ZIF-67 solution and stirred for $15 \mathrm{~min}$. Then $1 \mathrm{~mL}$ of $1.5 \mathrm{M}$ freshly $\mathrm{NaBH}_{4}$ was slowly added into the mixed solution under vigorous stirring for $1.5 \mathrm{~h}$. AuPt/ZIF-67 hybrids were obtained by centrifugation of the mixture with methanol. After that, the resulted AuPt/ZIF-67 was re-suspended in $10 \mathrm{~mL}$ methanol solution, then $0.5 \mathrm{~mL}$ $10 \mathrm{mM}$ of luminol was added to the AuPt/ZIF-67 solution and fully stirred for $10 \mathrm{~h}$. The mixed solution was centrifuged and washed to clean uncombined luminol. Lastly, the mixtures were dissolved in $1 \%$ chitosan solution and preserved at $4{ }^{\circ} \mathrm{C}$ for later use.

\section{Fabrication of $\mathrm{Ab}_{2}-\mathrm{MnO}_{2} @ \mathrm{CNTs}$ bioconjugates}

Firstly, $0.03 \mathrm{~g} \mathrm{KMnO}_{4}$ was deliquesced in $45 \mathrm{~mL}$ deionized water under magnetic stirring. Then $20 \mathrm{mg}$ CNTs, $1 \mathrm{M} \mathrm{H}_{2} \mathrm{SO}_{4}(2 \mathrm{~mL})$ and $1 \mathrm{~mL}$ PDDA were added to the above suspension and sonicated for $2 \mathrm{~h}$ with a time interval of $30 \mathrm{~min}$. CNTs and $\mathrm{KMnO}_{4}$ could be better dispersed by this ultrasonic treatment step to promote monomer adsorption on the CNTs wall. After ultrasonic treatment, a household microwave oven (Midea M1-L201B 800 W) was used to heat the mixed solution for $1 \mathrm{~min}$, and then cooled to RT. The heating and cooling processes were repeated 5 times. The precipitate was gathered by centrifuge and cleaning with deionized water several times. The final sediment $\left(\mathrm{MnO}_{2} @ \mathrm{CNTs}\right.$ composites) was dried at $100{ }^{\circ} \mathrm{C}$ overnight for later use. 
$\mathrm{MnO}_{2} @ \mathrm{CNTs}$ and $\mathrm{Ab}_{2}$ were coupled by electrostatic effect. $2 \mathrm{mg} \mathrm{MnO}_{2} @ \mathrm{CNTs}$ were added to $5 \mathrm{~mL}$ PBS $(0.01 \mathrm{M}, \mathrm{pH} 7.4)$ and stirred for $2 \mathrm{~h}$. After that, $600 \mu \mathrm{L}$ $10 \mu \mathrm{g} \mathrm{mL}^{-1} \mathrm{Ab}_{2}$ was added to the obtained $\mathrm{MnO}_{2} @ \mathrm{CNTs}$ solution for mixing and stirred at $4{ }^{\circ} \mathrm{C}$ for $12 \mathrm{~h}$. Then, $400 \mu \mathrm{L} 1.0 \mathrm{wt} \%$ BSA solution was introduced into above solution and continuously stirred at $4{ }^{\circ} \mathrm{C}$ for $1 \mathrm{~h}$ to prevent nonspecific binding, followed by centrifuging. The as-prepared $\mathrm{Ab}_{2}-\mathrm{MnO}_{2} @ \mathrm{CNTs}$ bioconjugates were resuspended in $1 \mathrm{~mL}$ PBS $(0.01 \mathrm{M}, \mathrm{pH} 7.4)$ at $4{ }^{\circ} \mathrm{C}$ for further use.

\section{ECL detection of RBP4}

The bare GCE was polished with 0.3 and $0.05 \mu \mathrm{m}$ alumina $\left(\mathrm{Al}_{2} \mathrm{O}_{3}\right)$ slurry for 3 min to acquire a mirror-like surface, followed by rinsing thoroughly with deionized water. Then, $5 \mu \mathrm{L}$ luminol@AuPt/ZIF-67 and $\mathrm{Ab}_{1}$ $\left(1 \mu \mathrm{g} \mathrm{mL}{ }^{-1}\right)$ suspension were incubated at $4{ }^{\circ} \mathrm{C}$ for least $2 \mathrm{~h}$, then washed with PBS $(0.01 \mathrm{M}, \mathrm{pH} 7.4)$ to remove unbounded $A b_{1}$. Next, the electrode was covered with $3 \mu \mathrm{L} 1.0 \mathrm{wt} \% \mathrm{BSA}$ solution to block the nonspecific binding for $45 \mathrm{~min}$ at $4{ }^{\circ} \mathrm{C}$. After rinsing with PBS $(0.01 \mathrm{M}, \mathrm{pH}$ 7.4) thoroughly, $10 \mu \mathrm{L}$ RBP4 of different concentrations were pipetted onto the AuPt/ZIF-67 decorated electrode surface then dried at $4{ }^{\circ} \mathrm{C}$ for least $1.5 \mathrm{~h}$. Finally, $5 \mu \mathrm{L}$ $\mathrm{Ab}_{2}-\mathrm{MnO}_{2} @ \mathrm{CNTs}$ bioconjugates was reacted with the modified electrode for $1 \mathrm{~h}$ to form the double antibody sandwich complex and further cleaned with PBS $(0.01 \mathrm{M}$, $\mathrm{pH}$ 7.4).

\section{Results and discussion}

\section{Characterization of ZIF-67 and AuPt/ZIF67}

The size, morphology and elemental composition of the as-synthesized nanomaterials were characterized by Transmission electron microscopy (TEM) and Scanning transmission electron microscopy-energy dispersive X-ray spectroscopy (STEM-EDS). As illustrated in Fig. 1A, B, the synthesized ZIF-67 showed a dodecahedral framework with a side length of about $500 \mathrm{~nm}$ and a very glossily surface. Moreover, after decorating with AuPt NPs, it was clear that the AuPt NPs were homogeneously dispersed on the surface of ZIF-67 (Fig. 1C, D) with an apex-to-apex diameter about $3 \mathrm{~nm}$. The element composition of AuPt/ZIF-67 was manifested by the elemental mappings of $\mathrm{C}, \mathrm{N}, \mathrm{O}, \mathrm{Au}, \mathrm{Pt}$, Co via STEM-EDS elemental mapping (Fig. 1E), which further proved that

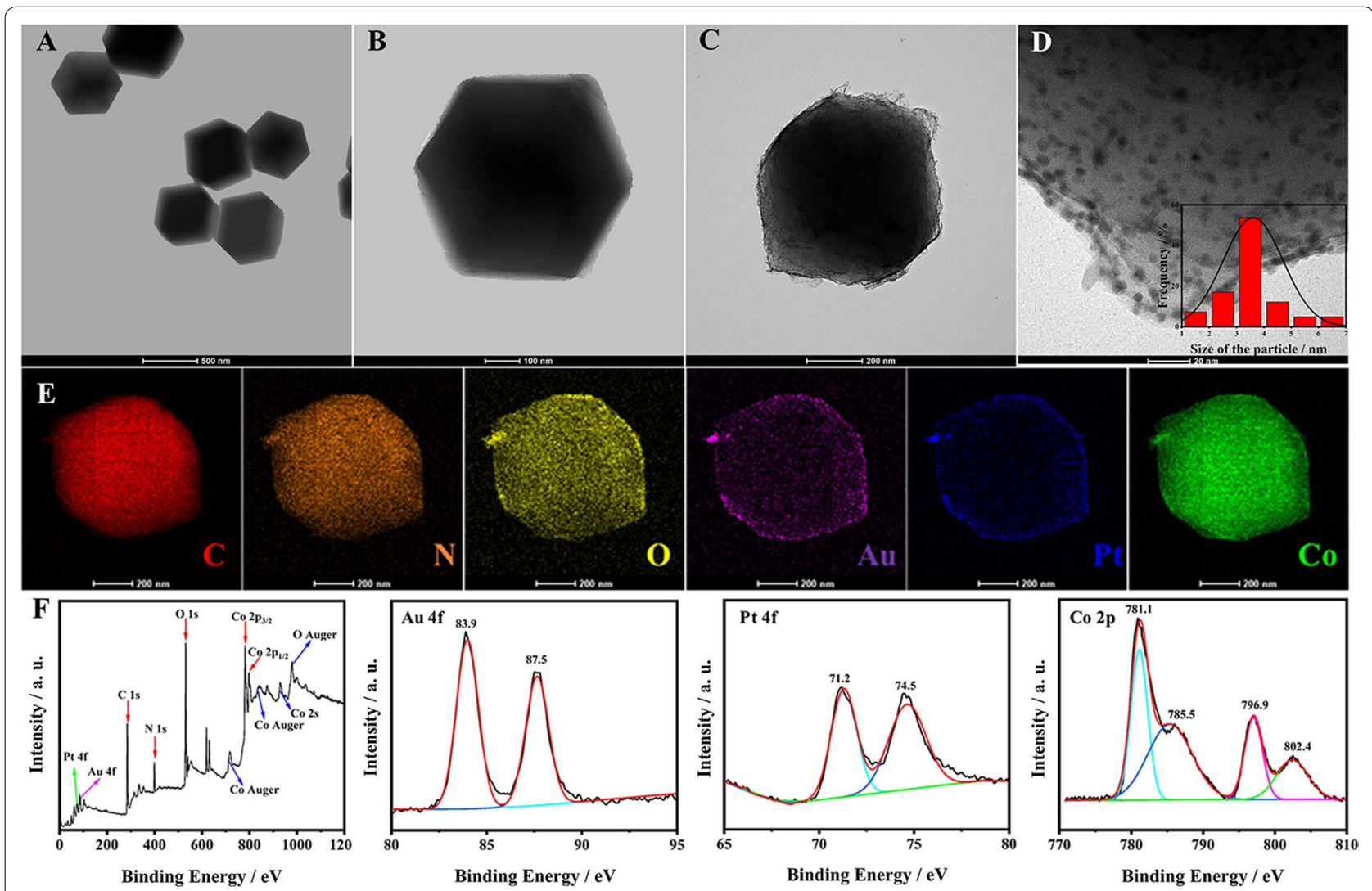

Fig. 1 A Low-magnifcation and B high-magnification TEM image of ZIF-67. C Low-magnification and D high-magnification TEM image of AuPt/ ZIF-67. The inset is the size distribution of AuPt NPS. E STEM-EDS mappings of AuPt/ZIF-67. F XPS high resolution spectra of AuPt/ZIF-67 
the surface of the ZIF-67 was uniformly dispersed AuPt bimetallic nanoparticles.

X-ray photoelectron spectroscopy (XPS) was used to further research the elemental composition and surface electronic state of the AuPt/ZIF-67 hybrids. As illustrated in Fig. 1F, the survey spectrum of AuPt/ZIF-67 indicated the presence of $\mathrm{Au}, \mathrm{Pt}, \mathrm{C}, \mathrm{N}, \mathrm{O}$ and $\mathrm{Co}$. The fitted peaks located at $83.9 \mathrm{eV}$ and $87.5 \mathrm{eV}$ were indexed to $\mathrm{Au} 4 \mathrm{f}$ peaks, indicating that the surface of the ZIF- 67 formed metallic gold [20]. The fitted peaks of Pt $4 \mathrm{f}$ at $71.2 \mathrm{eV}$ and $74.5 \mathrm{eV}$, demonstrated the forming of metallic Pt [34]. As shown in the high-resolution spectra of Co 2p, the fitted peaks at $781.1 \mathrm{eV}, 785.5 \mathrm{eV}, 796.9 \mathrm{eV}$ and $802.4 \mathrm{eV}$ could be assigned to metallic Co [35]. The XPS results were consistent with the STEM-EDS mapping results, indicating that the modified AuPt/ZIF-67 composites were successfully synthesized as expected.

To investigate the crystal structure, the ZIF-67 and AuPt/ZIF-67 composites were also characterized using $\mathrm{X}$-ray diffraction (XRD). As seen from Additional file 1: Fig. S1, the diffraction peaks of AuPt/ZIF-67 coincided exactly with simulated ZIF-67, demonstrating that the successful assembly of AuPt NPs on ZIF-67. Furthermore, the absence of XRD peaks from AuPt NPs could be probably attributed to the low NPs concentration as well as the small size of encapsulated AuPt NPs in the ZIF-67 framework.

\section{Characterization of CNTs and $\mathrm{MnO}_{2} @ C N T s$ nanocomposites} The morphology and structure of CNTs and $\mathrm{MnO}_{2} @$ CNTs were characterized with TEM, high-angle annular dark-field (HAADF) and energy-dispersive X-ray spectroscopy (EDS). From Fig. 2A, B, the as-prepared CNTs were uniform tubular structure with the average diameter about $8 \mathrm{~nm}$. After decorated with $\mathrm{MnO}_{2}$, it could be seen that $2 \mathrm{D} \mathrm{MnO}_{2}$ nanosheets in situ grows on the surface of CNTs with a typical layer-like structure. (Fig. 2D, E). Meanwhile, elemental analysis using EDS mapping was performed in Fig. 2G-K, the occurrence of $\mathrm{C}, \mathrm{N}, \mathrm{O}$, and $\mathrm{Mn}$ elements demonstrated that $\mathrm{MnO}_{2}$ nanosheets were uniformly dispersedly bonded onto the surface of CNTs.

The more detailed elemental composition and the oxidation state in the $\mathrm{MnO}_{2} @ \mathrm{CNTs}$ were characterized by XPS measurements, and the results of high-resolution XPS spectra of $\mathrm{C} 1 \mathrm{~s}, \mathrm{O} 1 \mathrm{~s}$, and Mn $2 \mathrm{p}$ are exhibited in Additional file 1: Fig. S2A. As shown in Additional file 1: Fig. S2B, the survey spectrum of $\mathrm{C} 1 \mathrm{~s}$ indicated three peaks at $284.3 \mathrm{eV}, 285.1 \mathrm{eV}, 286.1 \mathrm{eV}$ which consisted of the $\mathrm{C}=\mathrm{C}, \mathrm{C}-\mathrm{C}, \mathrm{C}=\mathrm{O}$ functional groups, respectively [36]. The high-resolution spectra of $\mathrm{O} 1 \mathrm{~s}$ in Additional file 1: Fig. S2C displayed two broad peak at $529.6 \mathrm{eV}$ and $531.1 \mathrm{eV}$, which could be correspond to two oxygen contributions [37]. There were two peaks located at $641.8 \mathrm{eV}$ and $653.2 \mathrm{eV}$ that could be assigned to $\mathrm{Mn} 2 \mathrm{p}_{3 / 2}$ and $\mathrm{Mn}$ $2 \mathrm{p}_{1 / 2}$ in Additional file 1: Fig. S2D. The peak separation of 11.4 eV between $\mathrm{Mn} 2 \mathrm{p}_{3 / 2}$ and $\mathrm{Mn} 2 \mathrm{p}_{1 / 2}$ in the $\mathrm{MnO}_{2} @$ $\mathrm{CNTs}$ demonstrated that Mn was present in the composite in the $\mathrm{Mn}^{4+}$ state [38].

XRD analysis was carried out to further explore the structure of $\mathrm{MnO}_{2} @ \mathrm{CNTs}$ nanocomposite material. As shown in Fig. $2 \mathrm{C}$, two stable diffraction peaks at $26^{\circ}$ and $42^{\circ}$ (marked with orange labels) indexed to the (002) and (101) crystal plane of CNTs (PDF card: JCPDS 65-6212) were observed. Furthermore, in the XRD pattern of $\mathrm{MnO}_{2} @ \mathrm{CNTs}$ (Fig. 2F), there were three broad peaks at around $12^{\circ}, 37^{\circ}$ and $66^{\circ}$ (marked with blue labels) could be obviously indexed to the (001), (111) and (312) crystal plane of $\mathrm{MnO}_{2}$ (PDF card: JCPDS 42-1317) except for the two diffraction peak of the CNTs pane [39]. All these characterizations demonstrated the successful synthesis of $\mathrm{MnO}_{2} @ \mathrm{CNTs}$.

\section{Enhancement and dual-quenched mechanism of the ECL immunosensor}

A possible ECL mechanism of the immunosensor was proposed as displayed in Scheme 1C. Luminol was oxidized to luminol anion $\left(\mathrm{Lu}^{-}\right)$which could transform into intermediate oxidation state of luminol (Lu.) under the scan of a positive potential. The synthesized AuPt/ZIF67 possessed superior peroxidase activity and excellent catalytic effect on $\mathrm{H}_{2} \mathrm{O}_{2}$, thus catalyzing $\mathrm{H}_{2} \mathrm{O}_{2}$ production of superoxide anion $\left(\mathrm{O}_{2}^{-}\right)$. Subsequently, $\mathrm{Lu}$ - would react with superoxide anion $\mathrm{O}_{2}{ }^{-\cdot}$ to generate the excited state of 3-aminophthalate $\left(3-\mathrm{AP}^{2-} *\right)$. Then, a heavily ECL signal was generated when the excited state $3-\mathrm{AP}^{2-} *$ returns to the ground state $3-\mathrm{AP}^{2-}$. In order to demonstrate the successful assembly of luminol on AuPt/ZIF-67 surface, the UV-Vis absorbance spectra of luminol, ZIF67, AuPt/ZIF-67 and luminol@AuPt/ZIF-67 were monitored (Additional file 1: Fig. S4). The UV-Vis absorbance spectrum of luminol@AuPt/ZIF-67 (curve d) could be observed at $355 \mathrm{~nm}$ and $600 \mathrm{~nm}$ with two distinct characteristic peaks corresponding to the absorbance of luminol (curve b) and ZIF-67 (curve a), respectively. To explore the ECL enhancement of luminol by the synthesized nanocomposites, we measured the ECL intensities of pure luminol (curve a), luminol@ZIF-67 (curve b) and luminol@AuPt/ZIF-67 (curve c) in solution (Fig. 3A). Compared with pure luminol, obvious enhancement of ECL signal was observed when luminol mixed with ZIF-67, and this signal was further enhanced after AuPt NPs introduced into the system. Hence, the synthesized nanozymes possessed superior catalytic performance attributed to ZIF-67 high peroxidase activity, which was consistent with the mechanism proposed above. 

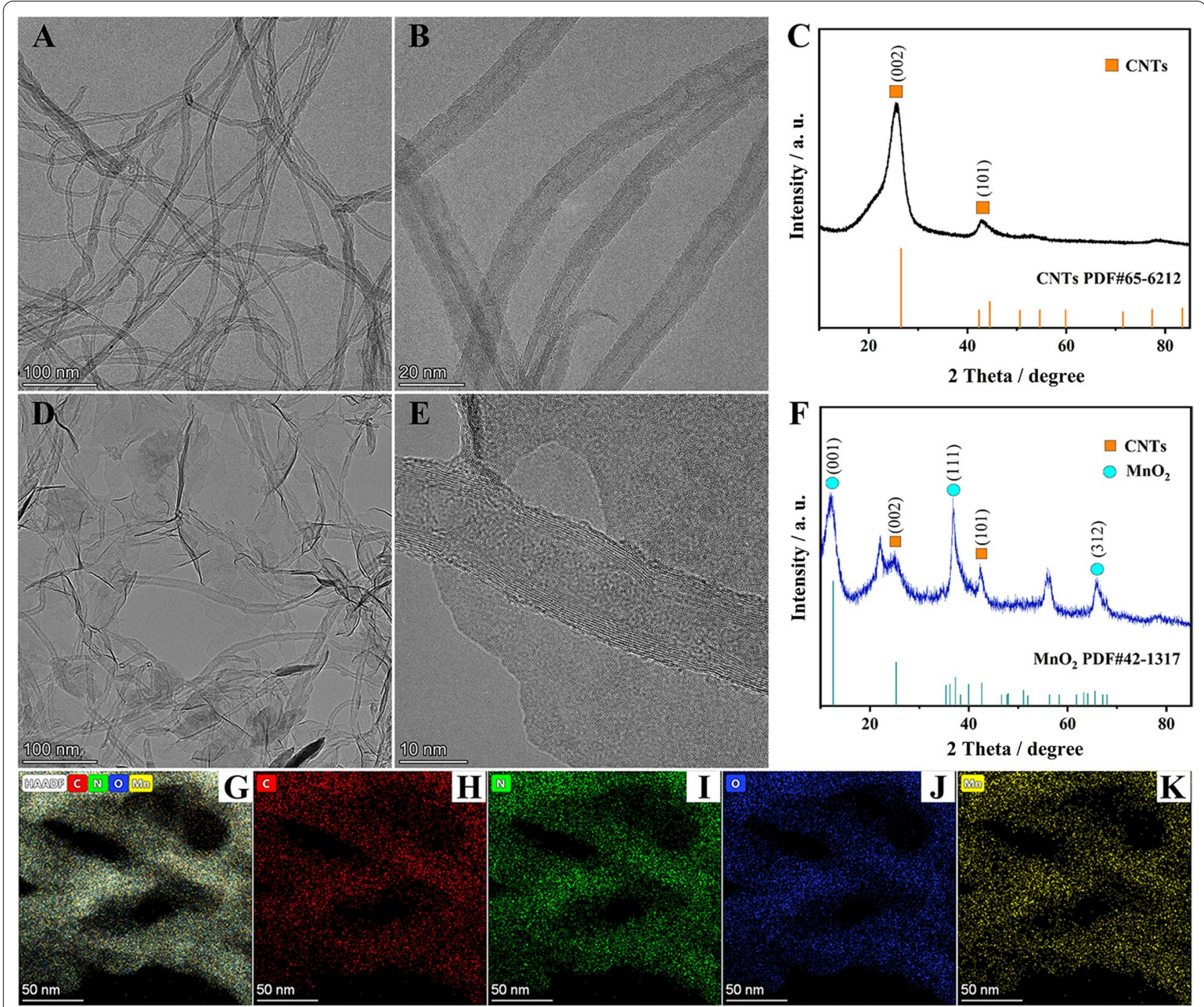

Fig. 2 A Low-magnification and $\mathbf{B}$ high-magnification TEM image of CNTs. D Low-magnification and $\mathbf{E}$ high-magnification TEM image of $\mathrm{MnO}_{2} @$ CNTs. XRD pattern of C CNTs and $\mathbf{F}$ MnO $_{2} @ C N T s$. G HAADF image and $\mathbf{H}-\mathbf{K}$ STEM-EDS elemental mappings of MnO $_{2} @ C N T s$ composites

After luminol combined with AuPt/ZIF-67, the assynthesized hybrids exhibited excellent catalytic performance with an obvious ECL signal boost Fig. 3B (curve a). When adding $20 \mathrm{mM}$ GSH to the solution, a significant quenching of the signal could be observed (curve b), which was due to the consumption of oxygen free radicals in the system. However, after adding $\mathrm{MnO}_{2} @ \mathrm{CNTs}$ quenchers to the substrate pool, the ECL signal further decreased though without GSH (curve c). The quenching mechanism may be that the absorption spectrum of $\mathrm{MnO}_{2}$ have proper overlaps with the ECL emission spectrum of luminol, which resulted in the quenching of ECL signal caused by ECL-RET [27]. Following the introduction of GSH in the luminol $/ \mathrm{H}_{2} \mathrm{O}_{2}$ coreactants system, the ECL signal was obviously attenuated (curve d) compared with the $\mathrm{MnO}_{2} @ \mathrm{CNTs} /$ luminol@AuPt/ZIF-67 modified electrode without GSH. The significant quenching effect mainly resulted from a large quantity of $\mathrm{Mn}^{2+}$ which was generated by GSH reduced $\mathrm{MnO}_{2}$, and then $\mathrm{Mn}^{2+}$ could consume the oxygen free radicals in the system and generate $\mathrm{MnOO}^{+}[40]$. The ECL immunosensor was successfully established based on the above dual-quench strategy which included ECL-RET between $\mathrm{MnO}_{2}$ and luminol, as well as consumption of ROSs by $\mathrm{Mn}^{2+}$.

\section{Characterizations of the prepared ECL immunosensor}

ECL dynamic curve and electrochemical impedance spectroscopy (EIS) were used to research the stepwise modification process of the ECL immunosensor. As displayed in Fig. 3C, when luminol@AuPt/ZIF-67 was 

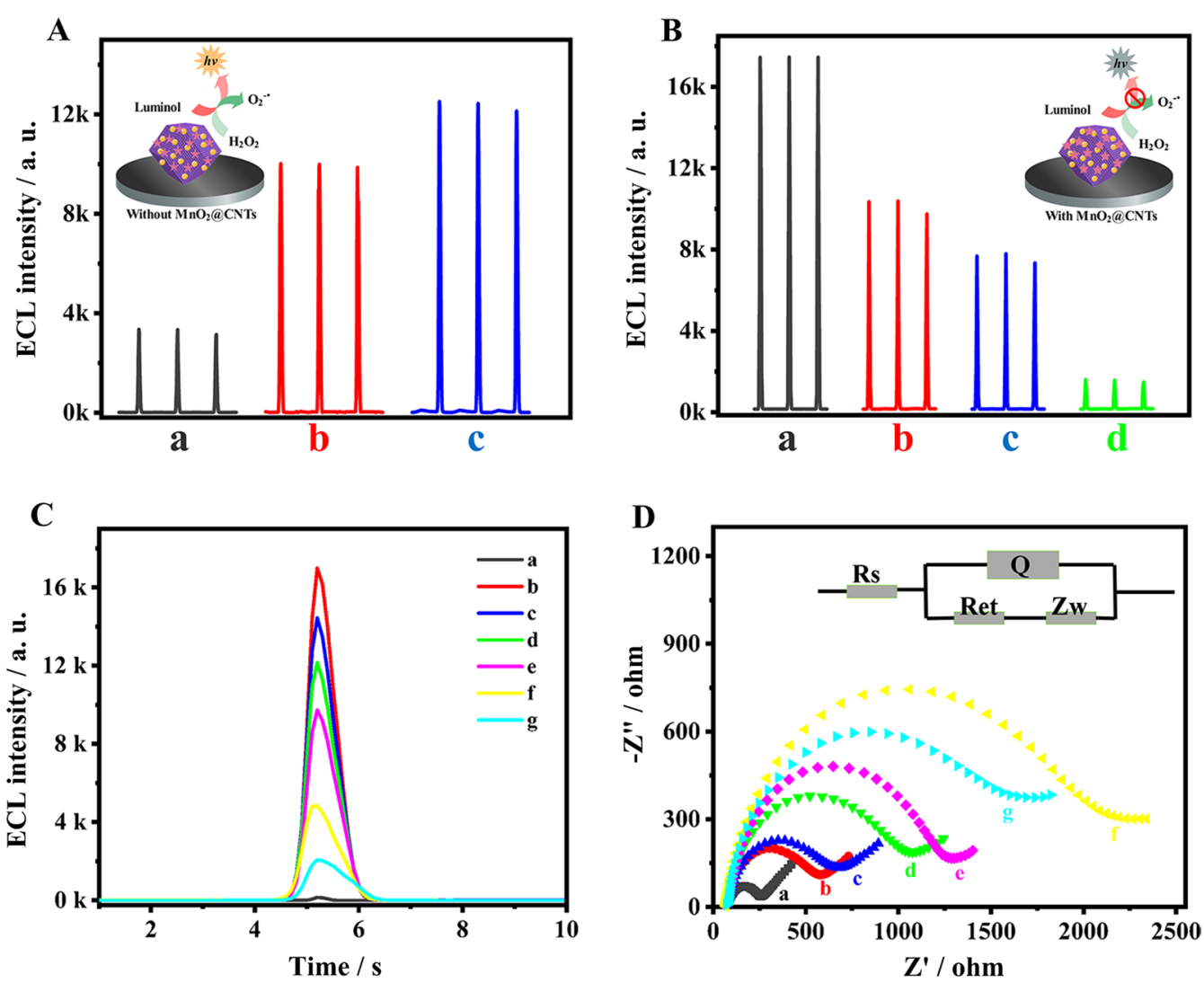

Fig. 3 A ECL behavior of pure luminol (a), ZIF-67@luminol (b), luminol@AuPt/ZIF-67 (c). B ECL behavior of luminol@AuPt/ZIF-67/GCE without (a) and with (b) GSH, $\mathrm{MnO}_{2} @ C N T s /$ luminol@AuPt/ZIF-67/GCE without (c) and with (d) GSH in $5 \mathrm{~mL}$ PBS (0.1 M, pH 8.0) containing $10 \mathrm{mM} \mathrm{H} \mathrm{H}_{2}$. C ECL curve and $\mathbf{D}$ EIS of bare GCE (a), after luminol@AuPt/ZIF-67 modification (b), after Ab, modification (c), after BSA blocking (d), after $10 \mathrm{ng} \mathrm{mL} \mathrm{mb}^{-1}$ RBP4 incubation (e), after $\mathrm{Ab}_{2}-\mathrm{MnO}_{2} @ \mathrm{CNTS}$ immobilization (f), and after adding $20 \mathrm{mM} \mathrm{GSH}(\mathrm{g})$, respectively measured in $5 \mathrm{~mL}$ PBS (0.1 M, pH 8.0) containing $10 \mathrm{mM} \mathrm{H}_{2} \mathrm{O}_{2}$ and in $5 \mathrm{mM} \mathrm{Fe}(\mathrm{CN})_{6}{ }^{3-/ 4-}$ containing $0.1 \mathrm{M} \mathrm{KCl}$ at $150 \mathrm{mV} \mathrm{s}^{-1}$. The inset was the equivalent circuit for EIS

decorated on the surface of GCE, the observed ECL signal was much higher than that of bare GCE (curve a via curve b), indicating that AuPt/ZIF-67 could boost the electron transfer to enhance ECL signal. With the following immobilization of $\mathrm{Ab}_{1}$ (curve c), BSA (curve d) and $10 \mathrm{ng} \mathrm{mL} \mathrm{mL}^{-1}$ RBP4 (curve e), the ECL signal decreased continuously because the deposited insulating proteins impeded the electron transfer between luminophor and $\mathrm{H}_{2} \mathrm{O}_{2}$. Moreover, the ECL signal was decreased dramatically (curve $\mathrm{f}$ ) when the modified electrode was incubated with $\mathrm{Ab}_{2}-\mathrm{MnO}_{2} @ \mathrm{CNTs}$ bioconjugates. After introducing $20 \mathrm{mM} \mathrm{GSH}$ to the system, the ECL strength further diminish (curve g) which was in accord with the above hypothesis.

As shown in Fig. 3D, the impedance curves of stepwise modification process were consistent with ECL results. High frequency semicircle of bare GCE was very small (curve a), indicating faster electron transfer on the electrode surface. When luminol@AuPt/ZIF-67 were modified on the electrode surface of GCE, high frequency semicircle slightly increased (curve b) which was related to the low conductivity of chitosan and ZIF-67. After being modified with $A b_{1}$ (curve c), BSA (curve d), RBP4 (curve e) and $\mathrm{Ab}_{2}-\mathrm{MnO}_{2} @ \mathrm{CNTs}$ (curve f), the resistance increased because of their negative charge that block the electron transfer rate. Since $\mathrm{MnO}_{2}$ could be consumed by GSH, thereby stripping the $\mathrm{MnO}_{2}$ from the $\mathrm{Ab}_{2}-\mathrm{MnO}_{2} @$ CNTs bioconjugates, the semicircle domain was decreased (curve g). Cyclic voltammetry (CV) curves of stepwise modification process (Additional file 1: Fig. S5) were consistent with ECL and EIS results, indicating the successful fabrication of modified electrode as expected.

\section{Optimization of the experimental conditions}

To obtain excellent analytical capability of the prepared immunosensor, the experimental conditions of the experiment were systematically optimized. Luminol ECL luminescence effect highly relies on $\mathrm{pH}$ value of solution, and only exhibits high efficiency in alkaline medium. The ECL signal sharply increased with the increasing $\mathrm{pH}$ value 
from 6.0 to 8.0 , and gradually decreased beyond $\mathrm{pH} 8.0$ (Fig. 4A). Hence, the $\mathrm{pH}$ value selected in this experiment was 8.0. The concentration of coreactant $\mathrm{H}_{2} \mathrm{O}_{2}$ was optimized in Fig. 4B. When $10 \mathrm{mM} \mathrm{H}_{2} \mathrm{O}_{2}$ was introduced to the detection solution, the ECL signal declined visibly with the further increasing of $\mathrm{H}_{2} \mathrm{O}_{2}$. Therefore, the optimized concentration of $\mathrm{H}_{2} \mathrm{O}_{2}$ was $10 \mathrm{mM}$, which would be used in subsequent experiments. Furthermore, the amount of $\mathrm{Mn}^{2+}$ which was generated by GSH reduced $\mathrm{MnO}_{2}$ played a key role in the quenching effect of the ECL signal. After introducing $20 \mathrm{mM} \mathrm{GSH}$ to the detection solution, the $\triangle E C L$ intensity (difference between RBP4/BSA/Ab 1 /luminol@AuPt/ZIF-67/GCE and $\mathrm{Ab}_{2}-\mathrm{MnO}_{2} @ \mathrm{CNTs} / \mathrm{RBP} 4 / \mathrm{BSA} / \mathrm{Ab}_{1} /$ luminol@AuPt/ZIF67/GCE) reached a platform (Fig. 4C). Consequently, $20 \mathrm{mM}$ GSH was selected for subsequent ECL detection to ensure optimal results.

The pulse potential also played an important role in ECL immunosensor and was optimized in the range 0.3$0.55 \mathrm{~V}$. As shown in Additional file 1: Fig. S6A, the ECL signal reached its highest value at $0.45 \mathrm{~V}$, which might be attributed to luminol can be electrochemically oxidized to luminol anion efficiently under a higher potential. The ECL signal showed an increasing to negative trend in the range -0.6 to $-0.35 \mathrm{~V}$ in Additional file 1: Fig. S6B. The maximum signal was obtained at $-0.55 \mathrm{~V}$, which may be because ECL initial potential of $-0.55 \mathrm{~V}$ has better diffusion control on the electrode surface.

\section{ECL detection performance of the immunosensor towards RBP4}

The efficacy of the prepared ECL immunosensor as a quantitative assay was investigated by varying the concentration of the RBP4 from 0.0001 to $100 \mathrm{ng} \mathrm{mL}^{-1}$ (curve $\mathrm{a}-\mathrm{j}$ in Fig. 5A). As seen in the calibration plots of Fig. $5 \mathrm{C}$, the correlation between the obtained $\mathrm{ECL}$ responses and the logarithm of the concentrations of RBP4 exhibited a good linear relationship in the range of $0.0001-100 \mathrm{ng} \mathrm{mL} \mathrm{m}^{-1}$. The linear equation was $\mathrm{I}=-539.15 \lg \mathrm{C}+2727.09$ (where I was ECL intensity and $\mathrm{C}$ was the concentration of RBP4) with the correlation coefficient $\left(R^{2}\right)$ of 0.9918 . The detection limit was calculated to be $43 \mathrm{fg} \mathrm{mL}^{-1}$ at a signal-to-noise ratio of $3(\mathrm{~S} / \mathrm{N}=3)$. In addition, compared with previously reported RBP4 detection methods (Additional file 1: Table S1), our method showed higher sensitivity and lower detection limits, attributing to the high electrocatalytic activity of AuPt/ZIF-67 nanozyme and robust signal quenching ability of the tailored $\mathrm{MnO}_{2} @ \mathrm{CNTs}$.

\section{Specificity, reproducibility, and stability of the immunosensor}

The specificity of the proposed immunosensor for RBP4 detection was evaluated to demonstrate its practicability. For this purpose, various substances such as alpha fetoprotein (AFP), follicle stimulating hormone (FSH), prolactin (PRL), bovine serum albumin (BSA) and glucose (Glu) were selected as potential interferences. The concentration of RBP4 was chosen as $1 \mathrm{ng} \mathrm{mL}^{-1}$, and all

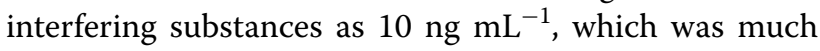
higher than the concentration of RBP4 selected for detection in the system. As shown in Fig. 5F, only when RBP4 and its mixture existed, the ECL signal could decrease significantly, which indicated that the immunosensor possessed an excellent selective response to RBP4 against other relevant biomolecules.

Meanwhile, we also evaluated the reproducibility of the fabricated immunosensor. The reproducibility of this immunosensor was obtained under the same reaction condition by measuring 6 identical electrodes for detecting $5 \mathrm{ng} \mathrm{mL}{ }^{-1} \mathrm{RBP} 4$. The measured relative standard deviation (RSD) was $1.31 \%$ (Fig. 5E). To make the
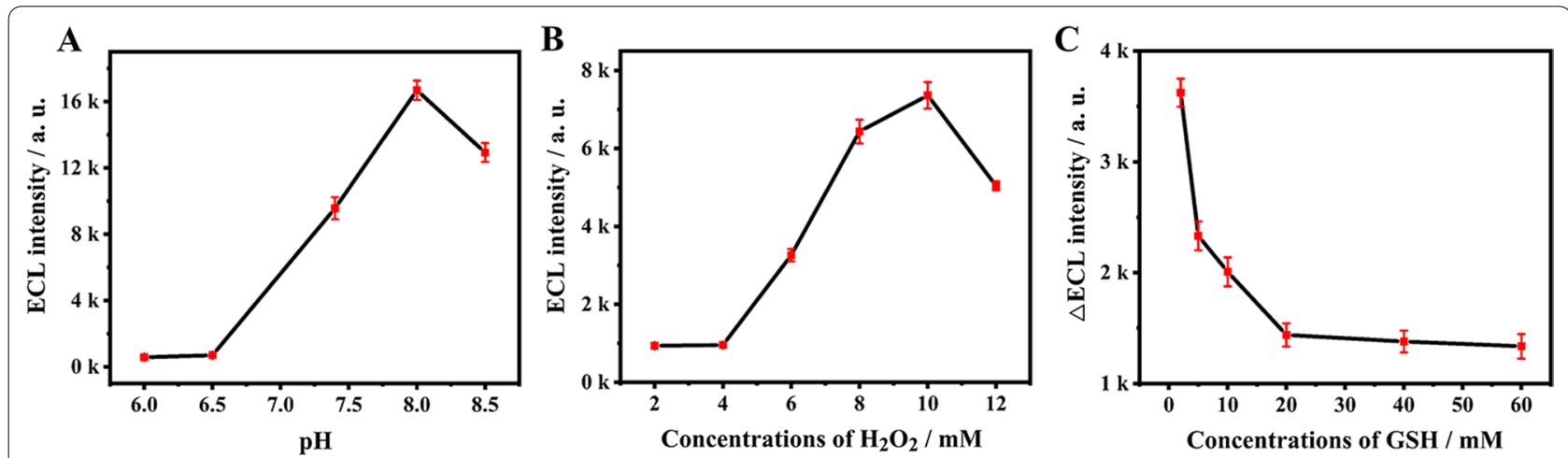

Fig. 4 Optimization of the detection conditions. A The effect of pH on the response of ECL intensity from luminol@AuPt/ZIF-67/GCE; $\mathbf{B}$ the concentrations of $\mathrm{H}_{2} \mathrm{O}_{2}$ in luminol/ $\mathrm{H}_{2} \mathrm{O}_{2}$ coreactants; $\mathbf{C}$ the effect of GSH concentrations on the ECL immunosensor for detection $5 \mathrm{ng} \mathrm{mL} \mathrm{m}^{-1} \mathrm{RBP} 4$ (error bars, $\mathrm{SD}, \mathrm{n}=3$ ) 


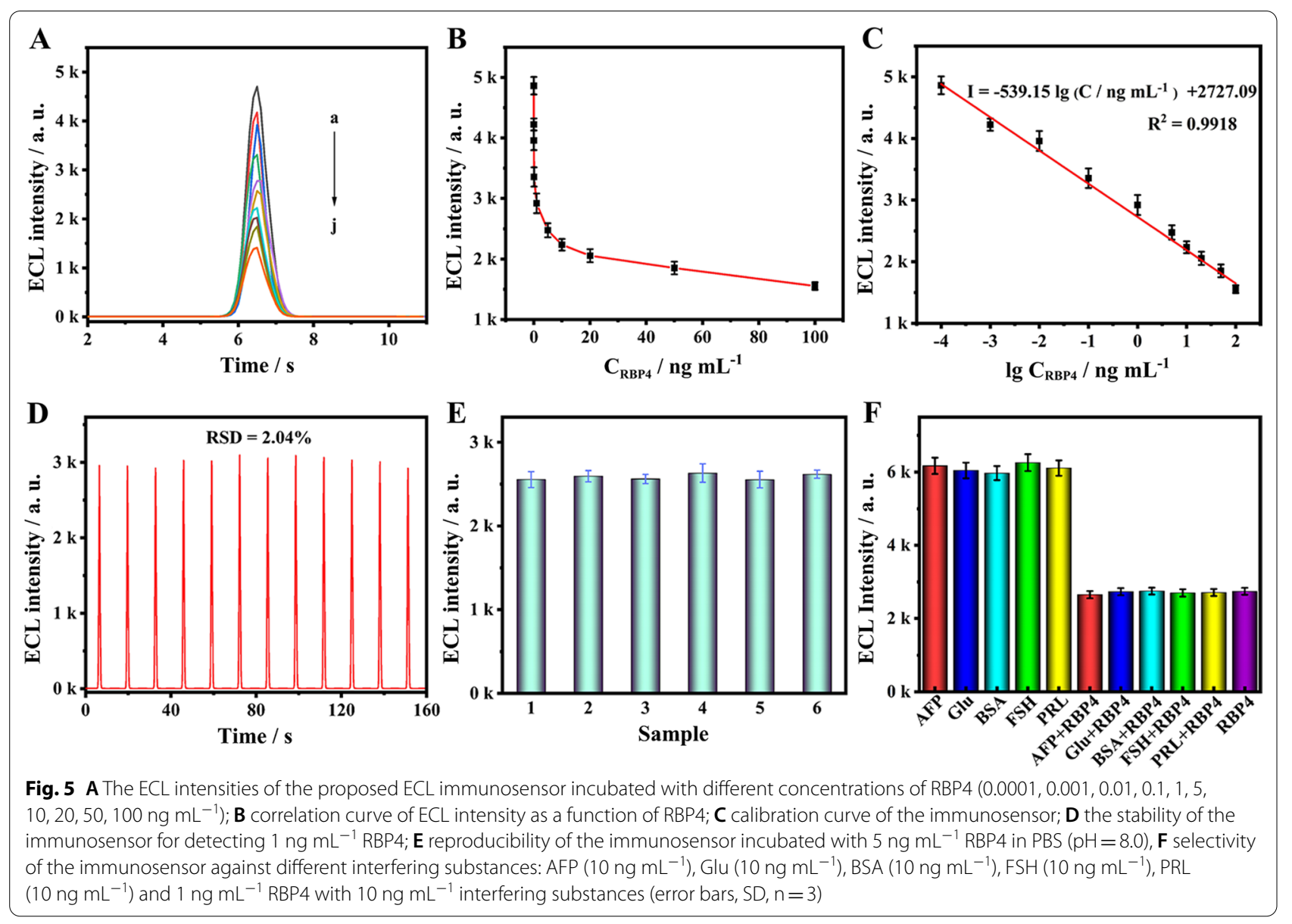

conclusions more credible, we have further optimized the reproducibility of the sensor at low $\left(0.001 \mathrm{ng} \mathrm{mL} \mathrm{m}^{-1}\right.$ RBP4), medium (5 $\mathrm{ng} \mathrm{mL}^{-1} \mathrm{RBP} 4$ ) and high concentrations (50 $\mathrm{ng} \mathrm{mL}{ }^{-1} \mathrm{RBP} 4$ ), the measured RSD were $5.09 \%, 1.31 \%$ and $4.53 \%$ respectively (Additional file 1 : Fig. S7), indicating good reproducibility of the proposed immunosensor.

The stability of the immunosensor was an important factor in evaluating whether it can be promoted in clinical practice. As illustrated in Fig. 5D, a steady ECL signal for RBP4 (1 $\left.\mathrm{ng} \mathrm{mL}^{-1}\right)$ within $160 \mathrm{~s}$ was observed with the average RSD of $2.04 \%$, indicating that the prepared immunosensor possessed excellent stability. Additionally, the ECL stability of the luminol@AuPt/ZIF-67/GCE was investigated in Additional file 1: Fig. S8, with the RSD of $0.14 \%$, which demonstrated that the sensing signal was reliable. Meanwhile, the proposed immunosensor was investigated by recording the ECL behavior storing for 10 days at $4{ }^{\circ} \mathrm{C}$. The ECL intensity decreased by $15.7 \%$ compared with the original ECL intensity after 10 days (Additional file 1: Fig. S9). These results suggested that the stability of the biosensor was satisfactory.

\section{Clinical sample analysis of the immunosensor}

To estimate the recovery efficiency of the immunosensor in complex serum matrices, the standard spikeand-recovery experiment was carried out. As shown in Table 1, the ECL immunosensor could be applied to detecting RBP4 in real human serum samples with a recovery of $96.60-111.32 \%$. All these results demonstrated that the proposed ECL immunoassay had potential applications in potentially detecting biomarkers of T2DM in serum.

\section{Conclusions}

In summary, based on luminol@AuPt/ZIF-67 hybrids and $\mathrm{MnO}_{2} @ \mathrm{CNTs}$ composites, a dual-quenched ECLRET immunosensor for ultrasensitive detection of RBP4 has been successfully constructed. The multi-function AuPt/ZIF-67 hybrids were employed as efficient peroxidase-like nanozymes, ECL donors and nanocarriers to load abundant luminol and antibodies, thus boosting the ECL performance of the luminol- $\mathrm{H}_{2} \mathrm{O}_{2}$ system. Subsequently, the amplified initial signal can be effectively 
Table 1 The recoveries of retinol binding protein 4 using the proposed ECL modified electrodes measured in $5 \mathrm{~mL}$ PBS (0.1 M, $\mathrm{pH}=8.0)$

\begin{tabular}{|c|c|c|c|c|c|}
\hline Sample & Addition (ng mL ${ }^{-1}$ ) & ECL intensity (a. u.) & Found $\left(\mathrm{ng} \mathrm{mL}^{-1}\right)$ & $\operatorname{RSD}(\%, n=3)$ & $\begin{array}{l}\text { Recovery } \\
(\%, n=3)\end{array}$ \\
\hline 1 & 50.00 & 1811.09 & 50.91 & 3.49 & 101.82 \\
\hline 2 & 25.00 & 1973.39 & 25.09 & 4.26 & 100.36 \\
\hline 3 & 5.00 & 2350.24 & 4.85 & 4.92 & 97.00 \\
\hline 4 & 0.50 & 2889.39 & 0.5566 & 2.74 & 111.32 \\
\hline 5 & 0.05 & 3428.54 & 0.0483 & 5.21 & 96.60 \\
\hline
\end{tabular}

quenched by $\mathrm{MnO}_{2} @ \mathrm{CNTs}$ composites through inhibiting peroxidase-like activity and ECL-RET strategy of luminol@AuPt/ZIF-67. Taking advantage of this dual signal quenching between luminol@AuPt/ZIF-67 hybrids and $\mathrm{MnO}_{2} @ \mathrm{CNTs}$ composites, the proposed ECL immunosensor for RBP4 detection achieved remarkable sensitivity, specificity and stability. In addition, the designed ECL immunosensor provides a promising platform not only for ultrasensitive detection of RBP4 to early monitor and diagnose T2DM, but also other biomarkers analysis. Obviously, future studies need to focus on simplifying detection procedure to promote clinical applications of ECL technique.

\section{Supplementary Information}

The online version contains supplementary material available at https://doi. org/10.1186/s12951-021-01020-1.

Additional file 1. Fig. S1. XRD pattern of Simulated ZIF-67, pure ZIF-67 and AuPt/ZIF-67. Fig. S2. XPS spectra of the MnO2@CNTs. Fig. S3. Typical catalytic oxidation reaction of 3, 3', 5, 5'-tetramethylbenzidine. Fig. S4. UV/Vis absorption spectra of ZIF-67, luminol, AuPt/ZIF-67 and luminol@ AuPt/ZIF-67. Fig. S5. Cyclic voltammetry (CV) curves of stepwise modification process. Fig. S6. Optimization of the reaction conditions. Reproducibility and stability of the immunosensor (Fig. S7, Fig. S8 and Fig. S9.).

Table S1. Comparison of ECL Immunosensor with other reported method.

\section{Acknowledgements}

Not applicable.

\section{Authors' contributions}

WG and SY carried out the experiments and wrote the manuscript. FZ drew schematic diagram. FT and JC analyzed experimental data. ZY and WY revised the manuscript. SD and RL provided funding and provided technical tools for experiments. All authors read and approved the final manuscript.

\section{Authors' information \\ Wei Gong is currently a master of Chongqing Medical University majoring in clinical medicine. His current research interests include the development of} biosensors and its clinical applications.

Suqing Yang is currently working in Chongqing Testing \& Inspection Center for Medical Devices. His research interests focus on immunosensors.

Fen Zhang is currently a master of Chongqing Medical University majoring in clinical medicine. Her current research interests include the development of biosensing strategy for clinical diagnosis.
Fengshun Tian is currently a master of Chongqing Medical University majoring in clinical medicine. His current research interests include the development of biosensors and DNA self-assembled.

Junman Chen is currently a Ph.D. of Chongqing Medical University majoring in clinical laboratory diagnosis. Her current research focus on multiple DNA self-assembled for molecular diagnosis.

Zhigang Yin is currently a master of Chongqing Medical University majoring in clinical medicine. His research interests focus on immunosensors.

Shijia Ding is currently a professor of Chongqing Medical University majoring in clinical laboratory diagnosis. His research interest is the development of biosensing strategy for clinical laboratory diagnosis.

Wei Yang is currently a Ph.D. of Chongqing Medical University majoring in clinical laboratory diagnosis. His current research interests include the development of biosensors and the application of the nanozymes.

Rong Luo is currently a professor of Chongqing Medical University majoring in clinical endocrinology. Her research interest is the development of biosensing strategy for clinical laboratory diagnosis.

Funding

This research was funded by the financial support from the National Science and Technology Major Project of the Ministry of Science and Technology of China (2018ZX10732202), and the National Natural Science Foundation of China (81873980).

\section{Availability of data and materials}

The datasets used and/or analysed during the current study are available from the corresponding author on reasonable request.

\section{Declarations}

Ethics approval and consent to participate

Not applicable.

\section{Consent for publication}

Not applicable.

\section{Competing interests}

The authors declare that they have no known competing financial interests or personal relationships that could have appeared to influence the work reported in this paper.

\section{Author details}

${ }^{1}$ Medical Examination Centre, The First Affiliated Hospital of Chongqing Medical University, Chongqing 400016, China. ${ }^{2}$ Department of Endocrinology, The First Affiliated Hospital of Chongqing Medical University, Chongqing 400016, China. ${ }^{3}$ Chongqing Testing \& Lnspection Center for Medical Devices, Chongqing 400016, China. ${ }^{4}$ Key Laboratory of Clinical Laboratory Diagnostics (Ministry of Education), College of Laboratory Medicine, Chongqing Medical University, Chongqing 400016, China. 
Received: 9 July 2021 Accepted: 30 August 2021

Published online: 08 September 2021

\section{References}

1. Hu Y, Ding M, Sampson L, Willett WC, Manson JE, Wang M, Rosner B, Hu FB, Sun Q. Intake of whole grain foods and risk of type 2 diabetes: results from three prospective cohort studies. BMJ. 2020;370:m2206.

2. Krentz NAJ, Gloyn AL. Insights into pancreatic islet cell dysfunction from type 2 diabetes mellitus genetics. Nat Rev Endocrinol. 2020;16(4):202-12.

3. Khunti K, Gavin J, Boulton A, Blickstead R, McGill M, Ceriello A, Raz I, Sadikot S, Wood D, Cos X, Kalra S, Das A, Espinosa López C, et al. The Berlin declaration: a call to improve early actions related to type 2 diabetes. Why is primary care important? Prim Care Diabetes. 2018;12(5):383-92

4. Campbell MD, Sathish T, Zimmet PZ, Thankappan KR, Oldenburg B, Owens DR, Shaw JE, Tapp RJ. Benefit of lifestyle-based T2DM prevention is influenced by prediabetes phenotype. Nat Rev Endocrinol. 2020;16(7):395-400.

5. Polonsky K. Retinol-binding protein 4, insulin resistance, and type 2 diabetes. N Engl J Med. 2006;354(24):2596-8.

6. Meex RCR, Watt MJ. Hepatokines: linking nonalcoholic fatty liver disease and insulin resistance. Nat Rev Endocrinol. 2017;13(9):509-20.

7. Fan J, Yin S, Lin D, Liu Y, Chen N, Bai X, Ke Q, Shen J, You L, Lin X, Li F, He $F$, Yan L, Chen C, Xia M. Association of serum retinol-binding protein 4 levels and the risk of incident type 2 diabetes in subjects with prediabetes. Diabetes Care. 2019;42(8):1574-81

8. Wang S, Wang J, Zhang R, Zhao A, Zheng X, Yan D, Jiang F, Jia W, Hu C, Jia W. Association between serum haptoglobin and carotid arterial functions: usefulness of a targeted metabolomics approach. Cardiovasc Diabetol. 2019;18(1):8.

9. Lee SJ, Park J-W, Kim I-A, Youn B-S, Gu MB. Sensitive detection of adipokines for early diagnosis of type 2 diabetes using enzyme-linked antibody-aptamer sandwich (ELAAS) assays. Sens Actuators B Chem. 2012;168:243-8.

10. Reyes-Garcia R, Rozas-Moreno P, Jimenez-Moleon JJ, Villoslada MJ, Garcia-Salcedo JA, Santana-Morales S, Munoz-Torres M. Relationship between serum levels of osteocalcin and atherosclerotic disease in type 2 diabetes. Diabetes Metab. 2012;38(1):76-81.

11. Wu J, Wang X, Wang Q, Lou Z, Li S, Zhu Y, Qin L, Wei H. Nanomaterials with enzyme-like characteristics (nanozymes): next-generation artificial enzymes (II). Chem Soc Rev. 2019;48(4):1004-76.

12. Wei H, Wang E. Nanomaterials with enzyme-like characteristics (nanozymes): next-generation artificial enzymes. Chem Soc Rev. 2013;42(14):6060-93.

13. Gao L, Zhuang J, Nie L, Zhang J, Zhang Y, Gu N, Wang T, Feng J, Yang D, Perrett S, Yan X. Intrinsic peroxidase-like activity of ferromagnetic nanoparticles. Nat Nanotechnol. 2007;2(9):577-83.

14. Abdelhamid H. Biointerface between ZIF-8 and biomolecules and their applications. Biointerface Res Appl Chem. 2021;11(1):8283-97.

15. El-Bery HM, Abdelhami HN. Photocatalytic hydrogen generation via water splitting using ZIF-67 derived $\mathrm{CO}_{3} \mathrm{O}_{4} @ \mathrm{C} / \mathrm{TiO}_{2}$.J Environ Chem Eng. 2021;9(4):105702.

16. Abdelhamid HN. Zeolitic imidazolate frameworks (ZIF-8, ZIF-67, and ZIF-L) for hydrogen production. Appl Organomet Chem. 2021. https:// doi.org/10.1002/aoc.6319.

17. Xie S, Ye J, Yuan Y, Chai Y, Yuan R. A multifunctional hemin@metalorganic framework and its application to construct an electrochemical aptasensor for thrombin detection. Nanoscale. 2015;7(43):18232-8.

18. Rui K, Zhao G, Lao M, Cui P, Zheng X, Zheng X, Zhu J, Huang W, Dou $S$, Sun W. Direct hybridization of noble metal nanostructures on 2D metal-organic framework nanosheets to catalyze hydrogen evolution. Nano Lett. 2019;19(12):8447-53.

19. Li X, Lu P, Wu B, Wang Y, Wang H, Du B, Pang X, Wei Q. Electrochemiluminescence quenching of luminol by CuS in situ grown on reduced graphene oxide for detection of $\mathrm{N}$-terminal pro-brain natriuretic peptide. Biosens Bioelectron. 2018;112:40-7.

20. Xing B, Zhu W, Zheng X, Zhu Y, Wei Q, Wu D. Electrochemiluminescence immunosensor based on quenching effect of $\mathrm{SiO}_{2} @ P D A$ on
$\mathrm{SnO}_{2} / \mathrm{rGO} / \mathrm{Au}$ NPs-luminol for insulin detection. Sens Actuators B Chem. 2018;265:403-11.

21. Xue $S$, Jing $P, X u W$. Hemin on graphene nanosheets functionalized with flower-like $\mathrm{MnO}_{2}$ and hollow AuPd for the electrochemical sensing lead ion based on the specific DNAzyme. Biosens Bioelectron. 2016;86:958-65.

22. Wang Y, Zhao G, Wang H, Cao W, Du B, Wei Q. Sandwich-type electrochemical immunoassay based on $\mathrm{CO}_{3} \mathrm{O}_{4} @ \mathrm{MnO}_{2}$-thionine and pseudoELISA method toward sensitive detection of alpha fetoprotein. Biosens Bioelectron. 2018;106:179-85.

23. Liu J, Meng L, Fei Z, Dyson P, Zhang L. On the origin of the synergy between the Pt nanoparticles and $\mathrm{MnO}_{2}$ nanosheets in Wonton-like 3D nanozyme oxidase mimics. Biosens Bioelectron. 2018;121:159-65.

24. Gao W, Liu Z, Qi L, Lai J, Kitte SA, Xu G. Ultrasensitive glutathione detection based on lucigenin cathodic electrochemiluminescence in the presence of $\mathrm{MnO}_{2}$ nanosheets. Anal Chem. 2016;88(15):7654-9.

25. Richter MM. Electrochemiluminescence (ECL). Chem Rev. 2004:104(6):3003-36

26. Fan $H$, Zhao Z, Yan G, Zhang X, Yang C, Meng H, Chen Z, Liu H, Tan W. A smart DNAzyme- $\mathrm{MnO}_{2}$ nanosystem for efficient gene silencing. Angew Chem Int Ed Engl. 2015;54(16):4801-5.

27. Li X, Sun X, Fan D, Yan T, Feng R, Wang H, Wu D, Wei Q. A ternary quenching electrochemiluminescence insulin immunosensor based on $\mathrm{Mn}^{2+}$ released from $\mathrm{MnO}_{2} @$ Carbon core-shell nanospheres with ascorbic acid quenching AuPdPt-MoS $\mathrm{MTiO}_{2}$ enhanced luminol. Biosens Bioelectron. 2019;142:111551.

28. Xue J, Jia Y, Yang L, Feng J, Wu D, Ren X, Du Y, Ju H, Wei Q. Etching triangular silver nanoparticles by self-generated hydrogen peroxide to initiate the response of an electrochemiluminescence sensing platform. Anal Chem. 2020;92(20):14203-9.

29. Song C, Li X, Hu L, Shi T, Wu D, Ma H, Zhang Y, Fan D, Wei Q, Ju H. Quench-type electrochemiluminescence immunosensor based on resonance energy transfer from carbon nanotubes and Au-nanoparticles-enhanced g- $\mathrm{C}_{3} \mathrm{~N}_{4}$ to $\mathrm{CuO} @$ Polydopamine for procalcitonin detection. ACS Appl Mater Interfaces. 2020;12(7):8006-15.

30. Deshmukh MA, Jeon JY, Ha TJ. Carbon nanotubes: an effective platform for biomedical electronics. Biosens Bioelectron. 2020;150:111919.

31. Wang Y, Wang S, Tao L, Min Q, Xiang J, Wang Q, Xie J, Yue Y, Wu S, Li X, Ding $H$. A disposable electrochemical sensor for simultaneous determination of norepinephrine and serotonin in rat cerebrospinal fluid based on MWNTs-ZnO/chitosan composites modified screen-printed electrode. Biosens Bioelectron. 2015:65:31-8.

32. Akhavan O, Ghaderi E, Shahsavar M. Graphene nanogrids for selective and fast osteogenic differentiation of human mesenchymal stem cells. Carbon. 2013;59:200-11.

33. Hu H, Guan B, Xia B, Lou XW. Designed formation of $\mathrm{CO}_{3} \mathrm{O}_{4} / \mathrm{NiCO}_{2} \mathrm{O}_{4}$ double-shelled nanocages with enhanced pseudocapacitive and electrocatalytic properties. J Am Chem Soc. 2015;137(16):5590-5.

34. Zhou X, Zhang W, Wang Z, Han J, Xie G, Chen S. Ultrasensitive aptasensing of insulin based on hollow porous $\mathrm{C}_{3} \mathrm{~N}_{4} / \mathrm{S}_{2} \mathrm{O}_{8}{ }^{2-}$ /AuPtAg ECL ternary system and DNA walker amplification. Biosens Bioelectron. 2020;148:111795

35. Dai L, Li Y, Wang Y, Luo X, Wei D, Feng R, Yan T, Ren X, Du B, Wei Q. A prostate-specific antigen electrochemical immunosensor based on $\mathrm{Pd}$ NPs functionalized electroactive Co-MOF signal amplification strategy. Biosens Bioelectron. 2019;132:97-104.

36. Guo J, Xu N, Wang Y, Wang X, Huang H, Qiao J. Bimetallic sulfide with controllable Mg substitution anchored on CNTs as hierarchical bifunctional catalyst toward oxygen catalytic reactions for rechargeable zinc-air batteries. ACS Appl Mater Interfaces. 2020;12(33):37164-72

37. Yuan C, Li J, Hou L, Yang L, Shen L, Zhang X. Facile template-free synthesis of ultralayered mesoporous nickel cobaltite nanowires towards high-performance electrochemical capacitors. J Mater Chem. 2012:22(31):16084-90.

38. Lee TH, Pham DT, Sahoo R, Seok J, Luu THT, Lee YH. High energy density and enhanced stability of asymmetric supercapacitors with mesoporous $\mathrm{MnO}_{2} @ \mathrm{CNT}$ and nanodot $\mathrm{MoO}_{3} @ \mathrm{CNT}$ free-standing films. Energy Storage Mater. 2018;12:223-31.

39. Yu P, Zhang X, Chen Y, Ma Y, Qi Z. Preparation and pseudo-capacitance of birnessite-type $\mathrm{MnO}_{2}$ nanostructures via microwave-assisted emulsion method. Mater Chem Phys. 2009;118(2-3):303-7. 
40. Liu C, Wang D, Zhang S, Cheng Y, Yang F, Xing Y, Xu T, Dong H, Zhang X. Biodegradable biomimic copper/manganese silicate nanospheres for chemodynamic/photodynamic synergistic therapy with simultaneous glutathione depletion and hypoxia relief. ACS Nano. 2019;13(4):4267-77.

\section{Publisher's Note}

Springer Nature remains neutral with regard to jurisdictional claims in published maps and institutional affiliations.
Ready to submit your research? Choose BMC and benefit from:

- fast, convenient online submission

- thorough peer review by experienced researchers in your field

- rapid publication on acceptance

- support for research data, including large and complex data types

- gold Open Access which fosters wider collaboration and increased citations

- maximum visibility for your research: over $100 \mathrm{M}$ website views per year

At BMC, research is always in progress.

Learn more biomedcentral.com/submissions 